\title{
Re-Engage: A Novel Nurse-Led Program for Survivors of Childhood Cancer Who Are Disengaged From Cancer-Related Care
}

\author{
Christina Signorelli, PhD ${ }^{1,2}$; Claire E. Wakefield, $\mathrm{PhD}^{1,2}$; Karen A. Johnston, MN-Nurs Prac ${ }^{1,2}$; Joanna E. Fardell, PhD ${ }^{1,2}$; \\ Jordana K McLoone, PhD 1,2; Mary-Ellen E. Brierley, BPsych(Hons) ${ }^{1,2}$; Maria Schaffer, BPsych(Hons) ${ }^{1,2}$; \\ Elysia Thornton-Benko, PhD ${ }^{3}$; Afaf Girgis, PhD'; W. Hamish Wallace, MD, FRACP5; and \\ Richard J. Cohn, MBBCh, FRACP1,2; on behalf of the BSU Implementation Group*
}

\section{ABSTRACT}

\begin{abstract}
Background: Survivors of childhood cancer often experience treatmentrelated chronic health conditions. Survivorship care improves survivors' physical and mental health, yet many are disengaged from care. Innovative models of care are necessary to overcome patient-reported barriers to accessing survivorship care and to maximize survivors' health. Methods: We piloted a novel survivorship program, called "Reengage," a distance-delivered, nurse-led intervention aiming to engage, educate, and empower survivors not receiving any cancerrelated care. Re-engage involves a nurse-led consultation delivered via telephone/online to establish survivors' medical history and needs. Participants completed questionnaires at baseline, 1 month postintervention, and 6-month follow-up. Results: A total of 27 survivors who had not accessed survivorship care in the last 2 years participated (median age, 31 years; interquartile range [IQR], 27-39 years); of which, $82 \%$ were at high-risk for treatment-related complications. Participation in Re-engage was high (75\%) and there was no attrition once survivors enrolled. At 1 month postintervention, $92 \%$ of survivors reported that Re-engage was "beneficial," which all survivors reported at 6-month follow-up. Survivors' overall satisfaction with their care increased from $52 \%$ before Re-engage to $84 \%$ at 1 month postintervention. Survivors' mean self-efficacy scores remained similar from baseline to 1 month postintervention ( $b=-0.33,95 \% \mathrm{Cl},-1.31$ to 0.65$)$, but increased significantly from baseline to 6-month follow-up $(b=1.64,95 \% \mathrm{Cl}$, $0.28-3.00$ ). At 6 -month follow-up, $73 \%$ of survivors showed an increase in health-related self-efficacy compared with baseline. Conclusions: Re-engage is a highly acceptable and feasible intervention and promotes health-related self-efficacy, which is integral to survivors being advocates for their own health. Further empirical work is needed to evaluate the long-term efficacy of Re-engage.

Trial registration: ACTRN12618000194268
\end{abstract}

J Natl Compr Canc Netw 2020;18(8):1067-1074 doi: 10.6004/jnccn.2020.7552

\footnotetext{
${ }^{1}$ Kids Cancer Centre, Sydney Children's Hospital, Sydney, NSW, Australia; ${ }^{2}$ School of Women's and Children's Health, UNSW Sydney, Sydney, NSW, Australia; ${ }^{3}$ Bondi Road Doctors, Bondi Junction, NSW, Australia; and ${ }^{4}$ Centre for Oncology Education and Research Translation, Ingham Institute for Applied Medical Research, South Western Sydney Clinical School, UNSW Sydney, Sydney, NSW, Australia; and ${ }^{5}$ Department of Hematology/Oncology, Royal Hospital for Sick Children, Edinburgh, United Kingdom.
}

*To view members of the BSU Implementation Group, see supplemental eAppendix 1 (available with this article at JNCCN.org).

\section{Background}

Most survivors of childhood cancer experience longterm health complications due to their cancer treatment, warranting lifelong care. ${ }^{1}$ Survivorship care provides opportunities for late effects prevention, screening, and proactive management. ${ }^{2}$ Ideally, survivorship care should be personalized and risk-based ${ }^{3,4}$; however, the optimal delivery of childhood cancer survivorship care is greatly debated. $^{5}$

Current models of survivorship care are inadequate. In many hospitals, the increasing number of survivors outweigh current service capacity, which relies primarily on oncologists. ${ }^{5,6}$ Many pediatric cancer centers are limited to major cities and do not offer lifelong survivorship care, necessitating transition to nonspecialized and adult services, a critical point at which many survivors disengage from care. ${ }^{6}$ These factors necessitate new models of care that empower survivors as guardians of their healthcare, ideally with healthcare coordination support. Nurses are increasingly recognized as key health professionals in survivorship care delivery, with preliminary evidence suggesting that nurse-led care is safe, acceptable, and feasible. ${ }^{7}$ Specialized nurse practitioners have the potential to optimize patient outcomes by equipping patients and families with the skills to manage their health needs, while maximizing the efficiency of healthcare delivery., ${ }^{8,9}$

As few as $42 \%$ of survivors of childhood cancer receive survivorship care in their adult years. ${ }^{10}$ Disengaged survivors miss out on opportunities for health education that can impact their health behaviors and healthcare use positively. They are also less likely to receive care from a health professional who is aware of their longterm cancer-related risks, ${ }^{11}$ leading to poorer physical and emotional outcomes compared with survivors who are engaged in specialized survivorship care. ${ }^{12}$

See JNCCN.org for supplemental online content. 
It can be challenging for survivors to manage their healthcare needs, given fluctuating health and frequent breaks in communication with health professionals. Managing the complexities of this changing state may be best suited to technology-enabled support that encourages self-management of care ${ }^{13}$ Educating survivors about their long-term risks is critical to promote engagement in survivorship care. Ideally, education should be an ongoing process into adulthood in order to accommodate survivors' information needs and preferences as they age. ${ }^{11,14}$

Although re-engaging survivors into active survivorship care is a priority, few studies have focused on this. To promote cancer-focused and risk-appropriate care, our team developed a new distance-delivered intervention, "Reengage," for survivors lost to follow-up. This nurse-led program aims to empower survivors to advocate for themselves in the healthcare system. Re-engage is purpose-designed to overcome survivor-, health professional-, and system-related barriers documented in the literature. ${ }^{11}$ Our pilot study aimed to assess the feasibility and acceptability of Re-engage and collect preliminary evidence of its impact on survivors' health-related self-efficacy, health behaviors, information needs, satisfaction with care, and emotional well-being.

\section{Methods}

The South Eastern Sydney Local Health District Human Research Ethics Committee approved this study (Ref:16/ 366). A detailed study protocol was published previously (eAppendix 2, available with this article at JNCCN.org). ${ }^{15}$

\section{Study Design}

We used a single group, pretest, and posttest design to assess the acceptability, feasibility, and potential efficacy of Re-engage.

\section{Participants}

We recruited survivors who: (1) were aged $>16$ years; (2) were diagnosed with at cancer $<18$ years of age; (3) had completed cancer treatment at Sydney Children's Hospital; (4) were at least 5 years postdiagnosis; and (5) had not received cancer-related survivorship care in the prior 2 years.

\section{Recruitment}

We identified survivors through electronic hospital records. The clinic nurse and oncologist also identified survivors. We mailed eligible participants an information package comprising a personalized invitation letter, information sheet, consent form, and opt-in/opt-out card. We classified survivors as uncontactable after 2 unsuccessful attempts to reach them via telephone or email 2 weeks after nonreceipt of opt-in/out cards. Participants who opted-in were provided information on how to borrow equipment for the study if needed (eg, telephone, computer/tablet, internet connection).

\section{Re-engage Intervention}

Re-engage is a distance-delivered live intervention requiring synchronous participation by survivors and the nurse via secure videoconferencing software or telephone, according to participants' preference. The Reengage intervention is described in Figure 1.

\section{Outcomes and Measures}

We collected data at baseline, 1 month postintervention, and 6-month follow-up via online/paper surveys according to participant preference (supplemental eTable 1).

We assessed acceptability in the 1 month postintervention survey, with questions evaluating satisfaction with program consultations length $(1=$ too long, 2 = just right, 3 = too short), program satisfaction based on the Youth Satisfaction Survey (eg, services needed/received: $1=$ strongly disagree; $5=$ strongly agree $),{ }^{16}$ satisfaction with care $(1=$ poor; $5=$ excellent), and perceived benefit and burden of the study $(1=$ not at all; $5=$ very much, plus open-ended questions). We determined a priori that Re-engage would be acceptable if $>80 \%$ of participants were satisfied with the program and feasible if we achieved at least $75 \%$ participation, $75 \%$ program compliance, and $<20 \%$ attrition. ${ }^{15}$

We assessed health-related self-efficacy using an 8-item measure based on a previously validated 3-item measure (eg, confidence asking doctor about concerns) combined with an additional 5 purpose-designed questions related to cancer survivorship (eg, confidence recognizing the signs of recurrence) ${ }^{17}$ Response options ranged from $1=$ not confident to $3=$ very confident. The summed score of each item formed a continuous composite score (range, 8-24), with higher scores representing better self-efficacy. The new 8-item measure showed reasonable reliability (Cronbach $\alpha=0.79$ ), and preliminary evidence of convergent construct validity with the original 3-item measure (baseline: $r=0.877$, $P<.01$; 1 month postintervention: $r=0.695, P<.01$; and 6-month follow-up: $r=0.878, P<.01$ ).

The secondary outcomes were survivors' health behaviors/beliefs, healthcare use, unmet information needs, satisfaction with care, and physical and mental well-being. We assessed survivor-reported reasons for not accessing survivorship care using an open-ended question.

\section{Risk and Needs Assessments}

We stratified participants using the Intensity of Treatment Rating scale 3.0 (ITR-3), which assigns survivors to 4 risk levels based on their cancer diagnosis, stage/risk, and 


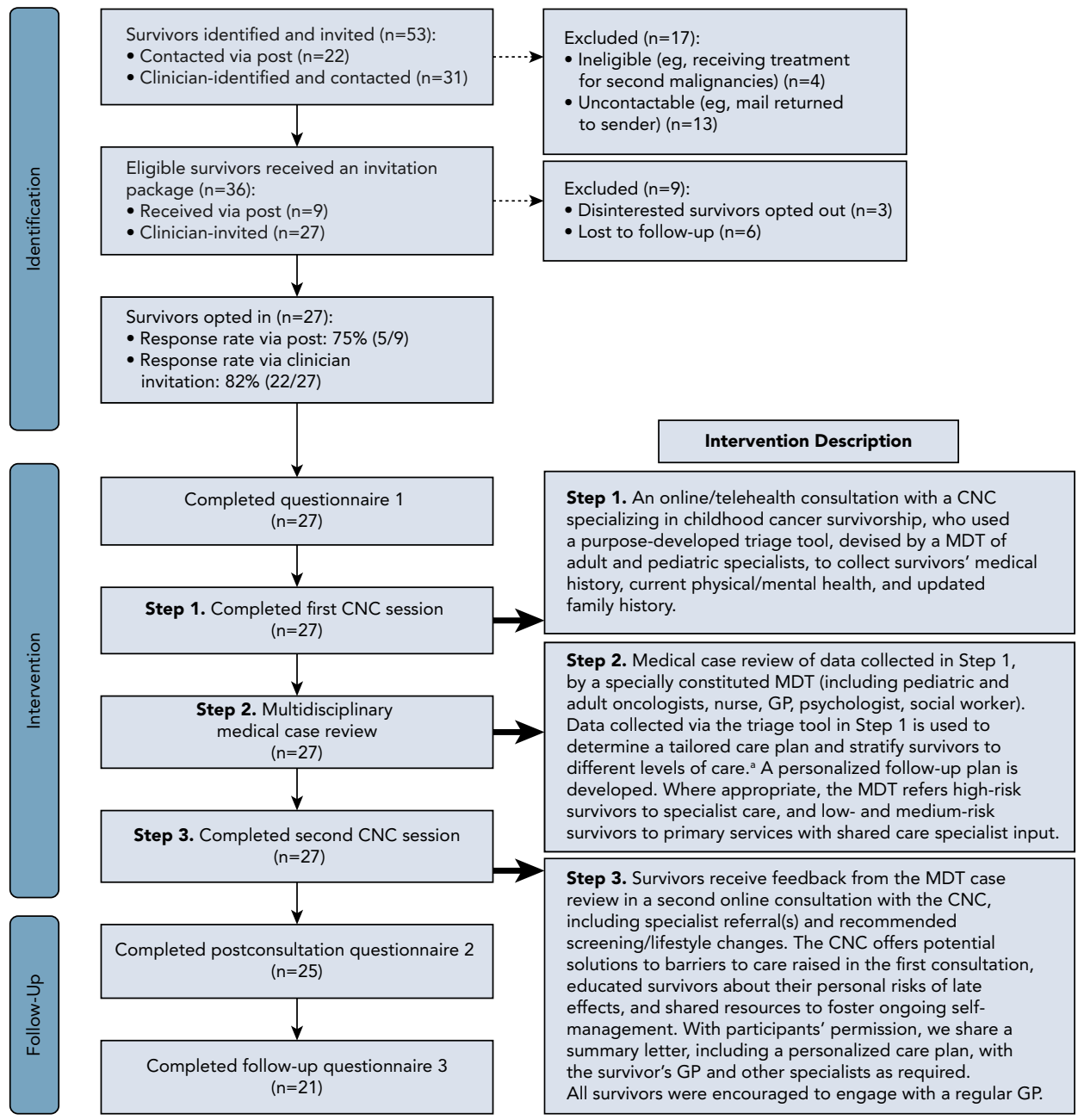

Figure 1. Recruitment flowchart.

Abbreviations: CNC, clinical nurse consultant; GP, general practitioner; MDT, multidisciplinary review.

aHigh-risk $=$ specialist/MDT care; low/medium-risk = primary services with specialist input where needed.

treatments received. ${ }^{18} \mathrm{~A}$ higher level (3 or 4 ) indicates higher treatment intensity and therefore higher risk of treatment-related conditions. The MDT also conducted an assessment of survivors' current medical and psychosocial needs using information collected with the triage tool.

\section{Data Analysis}

We analyzed quantitative data using SPSS Statistics, version 29 (IBM Corp), including descriptive statistics to summarize sample characteristics, analyze feasibility/acceptability, and summarize secondary outcomes. We compared selfefficacy scores preintervention and postintervention using multilevel models (MIXED function).

\section{Results}

Of 36 eligible and contactable survivors approached, 27 opted in to Re-engage (75\% response rate; Figure 1). All consenting survivors completed the full program (ie, there was no postenrollment attrition). Survey completion was high at 1 month postintervention $(n=25,93 \%)$ and at 6-month follow-up ( $\mathrm{n}=21,78 \%)$.

Among the participants, 59\% were male (Table 1) and median age at study enrollment was 31 years (interquartile range [IQR], 27-39 years). Using the ITR-3, $82 \%$ of survivors were classified as high risk (level 3 and 4). No survivors were low risk (level 1). Based on MDT assessments using information collected in the triage tool, $48 \%$ of survivors had medical or psychosocial needs prompting return to the survivorship clinic or specialist referral.

\section{Reasons for Disengagement}

All but 1 survivor reported reasons for not engaging in survivorship care (supplemental eFigure 1), with $35 \%$ reporting $>1$ reason, most commonly distance to clinic (30\%) and a low perceived need for survivorship care due to lack of health problems (30\%). 


\section{Table 1. Participant Characteristics ( $N=27)$}

\begin{tabular}{|c|c|}
\hline Characteristic & $\begin{array}{c}\text { Survivors } \\
\text { n (\%) }\end{array}$ \\
\hline Male survivors & $16(59)$ \\
\hline \multicolumn{2}{|l|}{ Self-identified ethnicity } \\
\hline Australian & $19(70)$ \\
\hline Not Australian & $7(30)$ \\
\hline \multicolumn{2}{|l|}{ Area of residence ${ }^{\mathrm{b}}$} \\
\hline Major city & $18(72)$ \\
\hline Regional/Remote & $7(28)$ \\
\hline \multicolumn{2}{|l|}{ Education } \\
\hline High school only & $10(37)$ \\
\hline Postschool (eg, university) & $17(63)$ \\
\hline Employed & $22(82)$ \\
\hline \multicolumn{2}{|l|}{ Income, AUD } \\
\hline$<\$ 60,000$ & $13(50)$ \\
\hline$\geq \$ 60,000$ & $13(50)$ \\
\hline \multicolumn{2}{|l|}{ Primary diagnosis } \\
\hline Leukemia & $10(40)$ \\
\hline Lymphoma & $4(16)$ \\
\hline Brain cancer & $6(24)$ \\
\hline Other & $5(20)$ \\
\hline \multicolumn{2}{|l|}{ Treatment received } \\
\hline Surgery & $15(60)$ \\
\hline Chemotherapy & $26(96)$ \\
\hline Radiation & $18(69)$ \\
\hline Bone marrow transplant & $5(28)$ \\
\hline Relapsed & $3(11)$ \\
\hline Second cancer & $3(11)$ \\
\hline \multicolumn{2}{|l|}{ ITR-3 risk leveld } \\
\hline Level 1 (low risk) & 0 \\
\hline Level 2 (medium risk) & $5(18)$ \\
\hline Level 3 (high risk) & $15(56)$ \\
\hline Level 4 (high risk) & $7(26)$ \\
\hline \multicolumn{2}{|l|}{ MDT needs assessment } \\
\hline Low & $6(22)$ \\
\hline Medium & $8(30)$ \\
\hline High & $27(48)$ \\
\hline
\end{tabular}

(continued)

\section{Feasibility}

The opt-in rate for clinician-identified survivors (82\%) was higher than for survivors recruited via post (75\%). A total of 13 survivors invited via post were deemed uncontactable (eg, package was returned to sender), and out-of-date details on medical records limited the success of this recruitment approach. A total of $44 \%$ of participating survivors opted in on receipt of a study
Table 1. Participant Characteristics ( $N=27$ ) (cont.)

\begin{tabular}{|lc|}
\hline Characteristic & $\begin{array}{c}\text { Survivors } \\
\mathbf{n}(\%)^{\mathbf{a}}\end{array}$ \\
\hline \begin{tabular}{l} 
Age, median \\
\cline { 2 - 2 } Interquartile range
\end{tabular} & 31.0 \\
\hline Age at diagnosis, mean (SD) & $27-39$ \\
\hline Range & $9.0(3.8)$ \\
\hline $\begin{array}{l}\text { Years since treatment completion, mean (SD) } \\
\text { Range }\end{array}$ & $0-16$ \\
\hline
\end{tabular}

Abbreviations: ITR-3, Intensity of Treatment Rating scale 3.0; MDT, multidisciplinary team.

aNumbers and percentages may not add up due to missing values and rounding errors.

bAccording to Area of Remoteness Index Australia (ARIA) classifications. 'Other diagnoses included osteosarcoma, rhabdomyosarcoma, and severe aplastic anemia.

${ }^{d} A$ higher rating indicates a higher treatment intensity based on diagnosis, stage, and treatment data.

invitation, and therefore did not require any follow-up calls. Among the remainder, the mean number of followup calls was 1.2 per survivor (range, 1-2). No participants required us to supply equipment for participation (eg, telephone, computer/tablet, internet connection). Two participants opted to have their consultations using videoconferencing and 25 selected the telephone. All consultations were completed with the patient alone, except in one case in which the survivor had cognitive impairments and was accompanied by his siblings.

Median length of the first consultation was 68 minutes (IQR, 45-90 minutes [SD, 29]), rated by $92 \%$ of survivors as "just right" $(n=23 / 25)$. Median length of the second consultation was 11 minutes (range, 7-15 minutes [SD, 5]), which all survivors rated as "just right." We did not observe any evidence of a difference in consultation length over the course of the study $(P=.420)$ or by diagnosis $(P=.185)$. However, consultation length was significantly longer in older participants $(P=.020)$.

\section{Acceptability}

Postintervention, $92 \%$ of survivors reported that the study was "beneficial" to them $(n=23 / 25)$. Four participants, 2 of whom did not go on to complete the 6 -month follow-up survey, rated the study as "a little bit" burdensome, which they attributed to the length of the surveys rather than the program itself. At 6-month follow-up, $100 \%$ of participants reported that the study was "beneficial" $(n=21)$ and 4 reported that it was "a little bit" burdensome, again attributed to the surveys.

Based on survey results at 1 month postintervention, Re-engage was shown to be highly acceptable, with $96 \%$ reporting satisfaction with the amount and quality of information delivered $(n=24 / 25)$ and indicating that they liked the help received $(n=24 / 25) ; 1$ survivor reported needing additional help (Figure 2). Preintervention, 
$52 \%$ of survivors were satisfied with their cancer-related care $(n=14 / 27)$ compared with $84 \%$ postintervention $(n=21 / 25)$.

Qualitatively, participants were highly supportive of the program. Six survivors reported benefiting from the information they received about their cancer history, healthcare needs, and recommended lifestyle changes. Five survivors reported that Re-engage provided them with access to specialist care, including a male neuroblastoma survivor aged 40 years, who stated that, "without things like [Re-engage], there is very little out there for long-term survivors." Two survivors indicated that Re-engage made them feel more "proactive" about their health, because it created an opportunity to seek care for unaddressed and sometimes suppressed concerns. They praised the remote delivery and ability to coordinate their future care more easily $(n=2)$.

\section{Preliminary Efficacy Evidence}

Survivors' mean health-related self-efficacy score before participating in the program was 17.16 . Compared with baseline, $44 \%$ of participants had an increase in selfefficacy scores by 1 month postintervention and $72 \%$ of participants had an increase in scores by 6 -month follow-up. There was strong evidence of a change in average self-efficacy over time $[F(2,21), 8.40 ; P=.002]$. Survivors' mean scores decreased from baseline to 1 month postintervention ( $b=-0.33 ; 95 \% \mathrm{CI},-1.31$ to 0.65 ), yet significantly increased from baseline to 6-month follow-up ( $b=1.64 ; 95 \%$ CI, 0.28-3.00) (Figure 3).

Survivors' engagement in risky behaviors (eg, smoking) and health behaviors (eg, exercise) remained similar preintervention, postintervention, and at follow-up (Table 2). Other health-related outcomes also remained stable, including quality of life and information needs. Survivors did appear more likely to report having a regular GP at 6-month follow-up (81\%) compared with baseline or 1 month postintervention (both 67\%), and reported brushing their teeth more regularly at 6-month follow-up (95\%) compared with baseline $(73 \%)$ and 1 month postintervention (79\%).

\section{Discussion}

Re-engage is a novel nurse-led e-health intervention developed to re-engage survivors into active follow-up and improve their health-related self-efficacy. Our data suggest that Re-engage is feasible to deliver and highly acceptable to survivors. Preliminary efficacy data seem promising for increasing survivors' self-efficacy and thus confidence in managing their own survivorship care into adulthood. Re-engage shows the potential to empower survivors in coordinating their complex care, and in the future may influence survivors' lifestyle, health beliefs, and overall physical and mental well-being to help maximize quality of life after surviving childhood cancer.

Survivors reported various reasons for not accessing cancer-related care, many of which align with internationally cited barriers. ${ }^{11}$ Distance is a prominent issue for survivors in Australia, where clinics are limited to major cities. ${ }^{6}$ Coupled with survivors' low perceived need for survivorship care ${ }^{19}$ and poor understanding of their personal risks and resulting surveillance needs, ${ }^{20}$ it is understandable that survivors cease to seek survivorship care. It is therefore encouraging that we experienced high uptake for Re-engage and no attrition, despite targeting the challenging population of disengaged survivors. Nevertheless, we encountered some challenges tracing some survivors such a long time after treatment completion, which is unsurprising given the highly mobile nature of this age group. ${ }^{21}$

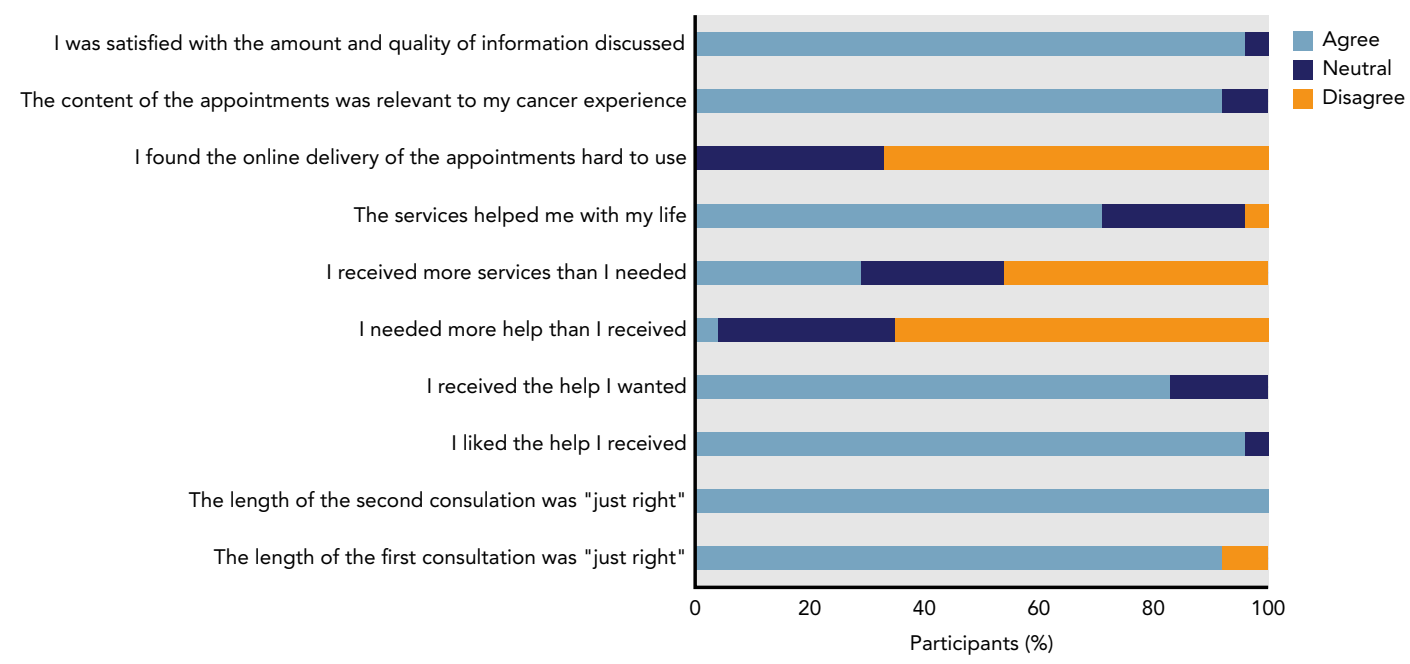

Figure 2. Participant ratings of acceptability of Re-engage at 1 month postintervention $(n=25)$. 
Survivors' openness to novel models of care is supported by the high acceptability ratings of Re-engage. Participants praised the nurse-led approach, despite survivors' well-established preference for oncologist-led models of care, ${ }^{22}$ supporting the potential for future nurse-led models in pediatric oncology. Nurse-led models have been used in adult cancer ${ }^{23}$ and other illnesses, ${ }^{24}$ but to date have been more acceptable among survivors of childhood cancer for supportive care rather than medical care. ${ }^{25}$ Offering specialist nurse-led care may be particularly important in the pediatric oncology context, in which survivors commonly report an attachment to their oncology team, leading to a reluctance to transition to adult healthcare, which may ultimately lead to disengagement. ${ }^{26}$

Preliminary evidence of the efficacy of Re-engage suggests that the program has the potential to improve survivors' health-related self-efficacy, empowering them to access the right care at the right time. It is not surprising that self-efficacy significantly increased at 6-month follow-up but not at 1 month postintervention. Given the complexity of survivors' needs, the longer period may have been necessary to enable action to address these needs, such as arranging an appointment at clinic or with the recommended specialists for further follow-up, or receiving treatment (eg, psychologic care) to resolve outstanding issues. Further longitudinal evaluation is needed to establish the long-term efficacy of Re-engage and drivers of self-efficacy, including clinical/ demographic factors and potentially modifiable drivers (eg, survivors' information needs).

A primary goal of survivorship care is to encourage healthy lifestyles in survivors in order to further reduce

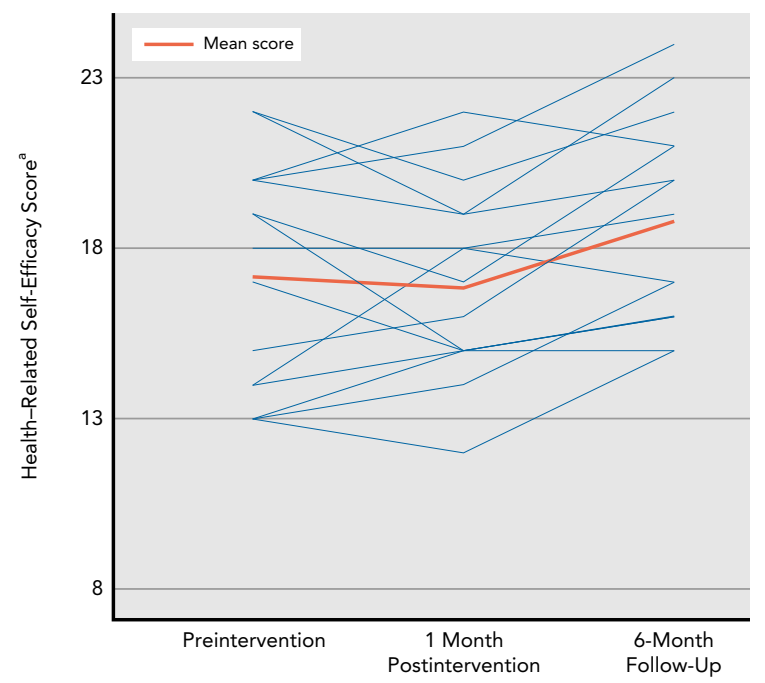

Figure 3. Mean self-efficacy scores at baseline, 1 month postintervention, and 6-month follow-up. The orange line is the mean score.

apossible scores: 8 to 24 their risk of late effects. Self-efficacy is also an important driver for behaviors such as information-seeking, careseeking, and risk-taking. For example, perceived control has been shown to be associated with survivorship care attendance, ${ }^{27}$ and positively associated with health behaviors such as physical activity. ${ }^{28}$ The opportunity for prevention and early detection reinforces the importance of (re)engaging, educating, and empowering survivors to receive appropriate lifelong care and minimize these adverse outcomes.

Re-engage did place a time burden on our staff. Our results have informed modifications to the execution of the next iteration of Re-engage that we are evaluating in an implementation trial. Manual completion of the triage tool during the first consultation was particularly timeconsuming. We are currently digitizing the tool, including drop-down options for common responses to speed completion. We will create a version that patients can complete before consultations, and will partially automate letters to survivors' GPs. We anticipate that these changes will reduce the overall nurse time/cost of delivering Re-engage. Streamlining the MDT meeting will further reduce intervention costs, such as including psychologist involvement for only high-risk cases. Cost is also a major barrier for survivors attending face-to-face appointments, and perpetuates nonattendance. ${ }^{11} \mathrm{Im}$ portantly, Re-engage incurs no direct costs for survivors, resulting in a significant cost-saving for them. Internationally, Re-engage may be partially reimbursable through the healthcare system (eg, Medicare Services in Australia and Canada, or some insurance companies in America). For the healthcare system, early detection of late effects in survivors participating in Re-engage has the potential to allow more cost-effective management of their care.

For $18 \%$ of Re-engage participants, review of their diagnosis/treatment alone suggested that they were at lower risk for serious/chronic health conditions (ie, ITR level 1 or 2). However, in $48 \%$ of cases, MDT assessment found physical and psychosocial issues that remained unaddressed despite receiving care in the community, highlighting the added value of the MDT assessments in recognizing risk factors that affect survivors' quality of life. For example, some survivors reported comorbidities that might exacerbate their risk of treatment-related late effects, such as obesity, which further increases their risk of cardiovascular disease and second cancers. ${ }^{29}$

\section{Strengths and Limitations}

Re-engage is one of the first attempts to address the barriers faced by many survivors who are disengaged from care, using an innovative nurse-led program and low-burden distance-delivered design. Our pilot suggests that Re-engage is effective in improving survivors' healthrelated self-efficacy in the medium term (up to 6 months). Our pilot did not include a control group, and it is possible 


\begin{tabular}{|c|c|c|c|}
\hline Total met information needs $(0-8, \uparrow=$ better $)$ & $4.5(2.83)$ & $4.7(2.82)$ & $5.1(2.98)$ \\
\hline Quality of life index $(0-1, \uparrow=$ better $)$ & $0.9(0.10)$ & $0.9(0.12)$ & $0.9(0.08)$ \\
\hline Anxiety score $(0-10, \downarrow=$ better $)$ & $3.6(2.53)$ & $3.2(3.08)$ & $3.5(2.21)$ \\
\hline Depression score $(0-10, \downarrow=$ better $)$ & $2.3(2.49)$ & $1.8(2.70)$ & $2.5(2.74)$ \\
\hline Distress score $(0-10, \downarrow=$ better $)$ & $2.2(2.17)$ & $1.9(2.61)$ & $2.4(2.37)$ \\
\hline \multicolumn{4}{|l|}{ Lifestyle factors } \\
\hline Hours of exercise (in the past week) & $2.8(3.52)$ & $4.6(10.49)$ & $5.6(10.44)$ \\
\hline \multicolumn{4}{|l|}{ Healthcare use and satisfaction, \% } \\
\hline Satisfied with cancer-related care & 52 & 84 & 81 \\
\hline Has a regular general practitioner & 67 & 67 & 81 \\
\hline Regular medication adherence & 93 & 92 & 84 \\
\hline \multicolumn{4}{|l|}{ Health behaviors, \% } \\
\hline Current smoker & - & 4 & 4 \\
\hline Brushes teeth twice daily, regularly & 73 & 79 & 95 \\
\hline Practices skin protection in the sun & 93 & 92 & 95 \\
\hline Consumes fruit daily & 41 & 46 & 26 \\
\hline
\end{tabular}

that self-efficacy may naturally improve over time. Few survivors opted for the videoconferencing option, instead choosing to complete their consultations via telephone. Further research to explore survivors' preferences for models delivered via e-health would be of value.

Our pilot sample size was small $(\mathrm{n}<30)$ and may overrepresent disengaged survivors who were already interested in re-engaging into survivorship care, particularly if they had previously received care from the nurse consultant, potentially biasing the results. We were not able to collect data about nonrespondents, including their reasons for nonparticipation. In addition, participants had varied primary cancer diagnoses, which may have impacted the results. Most of our participants were recruited opportunistically by clinicians who reviewed lists of patients who had not been to clinic in a long time or if survivors made contact first, potentially introducing some bias compared with systematically approaching all eligible survivors via hospital records. Compared with the Australian survivor population, ${ }^{30}$ our pilot sample had a similar proportion of men and women, and survivors diagnosed with leukemias, lymphomas, and brain cancer. However, our sample captured a larger proportion of survivors who were living rurally or remotely.
Given that most participants were Australian-born and all were English-speaking, the intervention should be trialed in non-English-speaking and culturally diverse populations, because they may face additional barriers to accessing care. Our study did, however, capture survivors with limited access to care, for example those living remotely and those who had moved interstate since being treated at Sydney Children's Hospital (28\%). Given the similarities in globally reported barriers to accessing survivorship care, ${ }^{11,31}$ Re-engage may be implemented in other international settings, with some modifications to suit local resources and practices. Further research investigating the effect of engagement in the healthcare system (eg, with their oncologist or GP) on survivors' satisfaction with care would be valuable.

\section{Conclusions}

This study shows that our novel distance-delivered, nurse-led Re-engage program is acceptable and feasible and illustrates early efficacy. Re-engage has the potential to empower survivors in coordinating their complex care, improving their long-term engagement, and increasing their satisfaction with care. Ultimately, Re-engage may also minimize the long-term burden associated with treatment of childhood cancer. 


\section{Acknowledgments}

We would like to thank Melody Huang for her valuable contribution to the study, and Mark Donoghoe for his advice regarding the statistical analysis. We are also grateful to the members of our Scientific Advisory Committee and Consumer Advisory Committee, who provided input in the design and ongoing conduct of the study. A special thanks also to the survivors who participated in Re-engage.

Submitted September 4, 2019; accepted for publication February 18, 2020.

Author contributions: Study concept, design, and protocol: All authors. Design of participant documents: Signorelli, Wakefield, Johnston, Fardell, Brierley, Schaffer, Thornton-Benko, Girgis, Wallace, Cohn. Data collection: Signorelli, Johnston, Fardell, Brierley, Schaffer, Thornton-Benko, Cohn. Data analysis: Signorelli. Manuscript preparation: All authors. Final approval of manuscript: All authors.
Disclosures: The authors have disclosed that they have not received any financial consideration from any person or organization to support the preparation, analysis, results, or discussion of this article.

Funding: This pilot study received financial support from the Australian Federation of Graduate Women Inc. Barbara Hale Fellowship, The Kids' Cancer Project, and a Cancer Council NSW Program Grant (PG16-02) with the support of the Estate of the Late Harry McPaul. The Behavioural Sciences Unit (BSU) is proudly supported by the Kids with Cancer Foundation. The BSU's survivorship research program is also funded by the Kids Cancer Alliance. Dr. Signorelli is supported by The Kids' with Cancer Project and is a Barbara Hale Scholar of 2017. Dr. Fardell is supported by The Kids' Cancer Project. Dr. Wakefield is supported by a Career Development Fellowship from the National Health and Medical Research Council of Australia (APP1143767). Dr. Girgis is supported by Cancer Institute NSW funding. None of the above funding bodies were involved in the study design or writing of the manuscript, nor were they involved in the analysis and interpretation of the results.

Correspondence: Christina Signorelli, PhD, Kids Cancer Centre, Sydney Children's Hospital, Level 1 South, High Street, Randwick, Sydney, NSW 2031, Australia. Email: c.signorelli@unsw.edu.au

\section{References}

1. Sadak KT, Neglia JP, Freyer DR, et al. Identifying metrics of success for transitional care practices in childhood cancer survivorship: a qualitative study of survivorship providers. Pediatr Blood Cancer 2017;64:e26587.

2. Shapiro CL. Cancer survivorship. N Engl J Med 2018;379:2438-2450.

3. Dixon SB, Bjornard KL, Alberts NM, et al. Factors influencing risk-based care of the childhood cancer survivor in the 21 st century. CA Cancer J Clin 2018:68:133-152.

4. Mayer DK, Alfano CM. Personalized risk-stratified cancer follow-up care: its potential for healthier survivors, happier clinicians, and lower costs. J Natl Cancer Inst 2019;111:442-448.

5. Tonorezos ES, Barnea D, Cohn RJ, et al. Models of care for survivors of childhood cancer from across the globe: advancing survivorship care in the next decade. J Clin Oncol 2018;36:2223-2230.

6. Signorelli C, Wakefield CE, McLoone JK, et al. Models of childhood cancer survivorship care in Australia and New Zealand: strengths and challenges. Asia Pac J Clin Oncol 2017;13:407-415.

7. Gates P, Krishnasamy M. Nurse-led survivorship care. Cancer Forum 2009; 33:175-178

8. Gardenier $\mathrm{D}, \mathrm{Ull}$ rich $\mathrm{T}$, Davis EL. Can primary care nurse practitioners provide survivorship care to cancer patients? J Nurse Pract 2018;14:228-229.

9. Garcia-Vivar C, Elizondo N, Ambrosio L. Primary care nursing is essential to fully implement survivorship care plans for long-term cancer survivors and their families. Cancer Nurs 2019;42:177-178.

10. Signorelli C, Wakefield CE, Fardell JE, et al. Perceptions of future health and cancer risk in adult survivors of childhood cancer: Implications for engagement in follow-up care. Cancer 2019:125:1008-1009.

11. Oeffinger KC, Wallace WHB. Barriers to follow-up care of survivors in the United States and the United Kingdom. Pediatr Blood Cancer 2006;46:135-142.

12. Signorelli $\mathrm{C}$, Wakefield CE, Fardell JE, et al. The impact of long-term follow-up care for childhood cancer survivors: a systematic review. Crit Rev Oncol Hematol 2017;114:131-138.

13. Peden C, Saxon L. Digital technology to engage patients: ensuring access for all. NEJM Catalyst. Accessed June 17, 2020. Available at: http:// catalyst.nejm.org/digital-health-technology-access/

14. Vetsch J, Fardell JE, Wakefield CE, et al. "Forewarned and forearmed": long-term childhood cancer survivors' and parents' information needs and implications for survivorship models of care. Patient Educ Couns 2017; 100:355-363.

15. Signorelli $C$, Wakefield CE, Johnston $K A$, et al. 'Re-engage' pilot study protocol: a nurse-led eHealth intervention to re-engage, educate and empower childhood cancer survivors. BMJ Open 2018;8:e022269.

16. Stüntzner-Gibson D, Koren PE, DeChillo N. The youth satisfaction questionnaire: what kids think of services. Fam Soc 1995;76:616-624.

17. Miller KA, Wojcik KY, Ramirez CN, et al. Supporting long-term follow-up of young adult survivors of childhood cancer: correlates of healthcare selfefficacy. Pediatr Blood Cancer 2017;64:358-363.
18. Kazak $A E$, Hocking $M C$, Ittenbach $R F$, et al. A revision of the intensity of treatment rating scale: classifying the intensity of pediatric cancer treatment. Pediatr Blood Cancer 2012:59:96-99.

19. Lee JL, Gutierrez-Colina A, Williamson Lewis R, et al. Knowledge of late effects risks and healthcare responsibility in adolescents and young adults treated for childhood cancer. J Pediatr Psychol 2019;44:557-566.

20. Lindell RB, Koh SJ, Alvarez JM, et al. Knowledge of diagnosis, treatment history, and risk of late effects among childhood cancer survivors and parents: the impact of a survivorship clinic. Pediatr Blood Cancer 2015;62:1444-1451.

21. Australian Bureau of Statistics. 4130.0.55.002 - Housing Mobility and Conditions, 2007-08. Accessed June 17, 2020. Available at: https://www. abs.gov.au/ausstats/abs@.nsf/mf/4130.0.55.002

22. Signorelli $\mathrm{C}$, Wakefield $\mathrm{CE}$, Fardell JE, et al. The role of primary care physicians in childhood cancer survivorship care: multiperspective interviews. Oncologist 2019;24:710-719.

23. Moore S, Corner J, Haviland J, et al. Nurse led follow up and conventional medical follow up in management of patients with lung cancer: randomised trial. BMJ 2002;325:1145

24. Watts $\mathrm{SA}, \mathrm{Gee} J, \mathrm{O}^{\prime}$ Day ME, et al. Nurse practitioner-led multidisciplinary teams to improve chronic illness care: the unique strengths of nurse practitioners applied to shared medical appointments/group visits. J Am Acad Nurse Pract 2009;21:167-172.

25. Goodenough B, Cohn RJ. Parent attitudes to audio/visual telecommunications in childhood cancer: an Australian study. Telemed J E Health 2004;10(Suppl 2)S-15-25.

26. Nandakumar BS, Fardell JE, Wakefield CE, et al. Attitudes and experiences of childhood cancer survivors transitioning from pediatric care to adult care. Support Care Cancer 2018;26:2743-2750.

27. Baenziger J, Roser K, Mader L, et al. Can the theory of planned behavio help explain attendance to follow-up care of childhood cancer survivors? Psychooncology 2018;27:1501-1508.

28. Keats MR, Culos-Reed SN, Courneya KS, et al. Understanding physical activity in adolescent cancer survivors: an application of the theory of planned behavior. Psychooncology 2007;16:448-457.

29. Moke DJ, Hamilton AS, Chehab L, et al. Obesity and risk for second malignant neoplasms in childhood cancer survivors: a case-control study utilizing the California Cancer Registry. Cancer Epidemiol Biomarkers Prev 2019;28:1612-1620

30. Youlden DR, Baade PD, Green AC, et al. The incidence of childhood cancer in Australia, 1983-2015, and projections to 2035. Med J Aust Med J Aust 2020;212:113-120.

31. Signorelli C, Wakefield C, McLoone JK, et al. Childhood cancer survivorship: barriers and preferences [published online November 11, 2019]. BMJ Support Palliat Care. doi: 10.1136/bmjspcare-2019-002001 